\title{
Validación de la Age Universal I-E Scale en sujetos mexicanos
}

\section{Validation of the Age Universal I-E Scale in Mexican Subjects}

Recepción: 04 Mayo 2015 | Aprobación: 09 Mayo 2016

\author{
Armando Rivera-Ledesma \\ Universidad de Londres, México \\ Sandra Zavala-Jiménez \\ Universidad de Londres, México \\ María Montero-López Lena \\ Universidad Nacional Autónoma de México, México \\ Rosa María García-Mendez \\ Universidad Latina, Coyoacán, México, México
}

**Tabasco 329. Colonia Roma, 06700.

Delegación Cuauhtémoc México D. F.

Correo electrónico:

armandoriveral@gmail.com;

zaji85@hotmail.com

***Facultad de Psicología. Av. Universidad

3004. Edif. D. 2do Piso Cub. 7. México D.

F. Correo electrónico:

monterol@unam.mx

****Pedro Henriquez Ureña No. 173, Col.

Los Reyes, Coyoacan, México, D.F.,

04330 Correo electrónico:

rmgarcia@unila.edu.mx

\section{RESUMEN}

Se evalúan las propiedades psicométricas de la Escala I-E-12 en tres muestras de sujetos mexicanos: religiosos $(\mathrm{N}=78)$, no religiosos $(\mathrm{N}$ = 148) y una muestra mixta $(\mathrm{N}=226)$. Mediante análisis factorial exploratorio se obtuvo una estructura compuesta por tres factores, siendo la estructura de grupos religiosos la que explicó el $63.5 \%$ de la varianza con una consistencia interna alfa de entre 0.70 y 0.88 para la escala total y sus diferentes factores. La escala evaluada en sujetos mexicanos muestra resultados consistentes en buena medida con lo reportado por Simkin y Etchezahar (2013) en la exploración de la I-E 12, en el contexto argentino.

\section{Palabras clave}

Age Universal I-E Scale, orientación religiosa, validación, México.

\begin{abstract}
The work evaluates the psychometric properties of the scale I-E-12 in three Mexican samples: religious $(\mathrm{N}=78)$, non religious $(\mathrm{N}=148)$ and composite sample $(\mathrm{N}=226)$. After the exploratory factor analysis applied to each of the samples, there was a structure composed of three factors being the structure of religious groups which he explained the $63.5 \%$ of the variance with an alpha internal consistency between 0.7 and 0.88 for the full scale and its various factors. The scale assessed in Mexican subjects shows consistent results in good measure to those reported by Simkin and Etchezahar (2013), in the exploration of the I-E-12 in the argentine context.

Keywords

Age Universal I-E Scale, religious orientation, validation, Mexico.
\end{abstract}

Para citar este artículo: Rivera-Ledesma, A., Zavala-Jiménez, S., Montero-López, M. , \& García-Méndez, R.M. (2016). Validación de la Age Universal I-E Scale en sujetos mexicanos. Universitas Psychologica, 15 (4). http://dx.doi.org/10.1114/Javeriana.up sy15-4.vaus 
La Age Universal I-E Scale ([I-E-12]; Maltby, 1999) deriva de la Escala de Orientación Religiosa (ROS) desarrollada por Allport y Ross (1967). La ROS fue construida dentro de un contexto cultural judío-cristiano (Allport \& Ross, 1967), introduciendo en el campo de la Psicología de la Religión los constructos de religiosidad intrínseca y religiosidad extrínseca. La religiosidad intrínseca fue definida como el resultado de una motivación última y trascendente, fundamental para el sujeto. Dentro de esta orientación, de acuerdo con Allport y Ross, cualquier otra necesidad por importante que pudiese resultar sería relegada o sujetada armónicamente al motivo fundamental de la vida del sujeto: sus valores religiosos. Este realizaría esfuerzos cotidianos importantes para internalizar, para incorporarse activamente dichos valores y seguirlos.

Por otro lado, y en una tesitura distinta, la religiosidad extrínseca implicaría una motivación utilitaria subyacente a la conducta religiosa; aquí la religión juega un papel periférico en la vida del sujeto, que se involucra en creencias, conductas y actitudes religiosas en la medida en que le permiten alcanzar metas como confort emocional, sentimiento de protección, adquisición de estatus y aprobación social, distracción, autojustificación, etc. En esta escena, la relación que el sujeto establece con lo divino queda mediatizada por sus propios intereses como condición básica de aproximación.

Esencialmente, la diferencia entre la orientación intrínseca y extrínseca es que la primera busca activamente un cambio interior en la dirección demarcada por lo divino o por la doctrina religiosa, en tanto que la segunda no asumiría dicho cambio y sí, una condición utilitaria de la religiosidad. De acuerdo a esto, el énfasis parecería estar en el uso de la religiosidad : no como medio para religarse a lo divino, sino como recurso de afrontamiento, como medio para acceder a lo mundano, por oposición a lo espiritual. Así, los valores extrínsecos son de carácter instrumental y utilitario. Más tarde, Kirkpatrick (1989) propondría que la orientación extrínseca podría ser de orden social, cuando se enfoca en la búsqueda de amigos en la iglesia, o en el pasar tiempo y disfrutar estando con ellos, como es dado identificar al analizar los ítems de este factor en el instrumento propuesto por el investigador; igualmente, la religiosidad extrínseca incluiría, de acuerdo con el autor un factor de orden personal cuando el sujeto busca obtener alivio, protección, paz y felicidad. Allport y Ross (1967) propondrían que "(...) la mayoría de las personas que profesan una religión caen en un continuum entre esos dos polos" (p. 434), entre la orientación intrínseca y la orientación extrínseca y que los casos puros donde imperase uno de los dos tipos ideales de religiosidad serían muy raros. Operacionalmente, este planteamiento supondría que entre ambas orientaciones religiosas existiría una asociación inversa, importante y significativa, lo cual se ha discutido ampliamente (Burris, 1999), reportándose asociaciones entre las subescalas intrínseca y extrínseca (social y personal) que no apoyan tal hipótesis. Maltby et al. (2010), por ejemplo, reportaron correlaciones significativas de -0.05 y 0.14 para la orientación extrínseca personal y la social, respectivamente; Maltby (2002), -0.04 y 0.08 y Simkin y Etchezahar (2013), 0.69 y 0.39, en un contexto latinoamericano, asociaciones altas $\mathrm{y}$ significativas, pero directas. Se ha sugerido que en realidad no se trata de un continuo de opuestos entre ambas orientaciones, sino de dos dimensiones independientes (Batson, Schoenrade, \& Ventis, 1993 citados por NúñezAlarcón, Moreno-Jímenez, \& Moral-Toranzo, 2011).

Ahora bien, la ROS ha sido evaluada y modificada sucesivamente en contextos anglosajones por investigadores tales como Gorsuch y Venable (1983), Kirkpatrick (1989), Leong y Zachar (1990), entre otros, llevando la escala original de 20 ítems propuestos por los Allport y Ross (1967), para la medición de los constructos de religiosidad intrínseca y religiosidad extrínseca, a 12 ítems (Escala IE-12 de Kirkpatrick, 1989), divididos en tres factores evidenciados por el análisis factorial que parecen medir Religiosidad intrínseca, 
Religiosidad extrínseca personal y Religiosidad extrínseca social.

Esta última versión de Kirkpatrick (1989), la Escala I-E 12, ha sido adaptada al español en sujetos argentinos por Simkin y Etchezahar (2013) y explorada y confirmada mediante análisis factorial en una muestra no probabilística de estudiantes autodeclarados religiosos, aportando evidencia con respecto a la estructura trifactorial de la I-E-12, con buenas propiedades psicométricas en cuanto a consistencia interna y validez.

La ROS ha demostrado su utilidad en la investigación anglosajona, y muy especialmente en estudios sobre el prejuicio existente hacia grupos vulnerables. Sin embargo, el empleo de esta escala se encuentra limitado en Latinoamérica en virtud de la inexistencia de suficientes estudios tendientes a la exploración de las propiedades psicométricas de la escala en contextos latinos. Por ello, el objetivo de este trabajo fue evaluar las propiedades psicométricas de la I-E-12, en tres muestras de sujetos mexicanos: religiosos, no religiosos y en una muestra mixta (con ambos grupos), a partir de la versión adaptada en sujetos argentinos por Simkin y Etchezahar (2013).

\section{Método}

La muestra fue seleccionada mediante un procedimiento no probabilístico intencional, en un contexto universitario laico, conformado por diferentes profesiones. En total se aplicaron 300 cuestionarios, considerando sujetos que manifestaron su interés en participar en la investigación mediante la firma de su consentimiento informado. De los 300 cuestionarios iniciales se descartaron 74 por razones atribuibles al llenado de los reactivos, quedando una muestra final de 226 sujetos con una edad media de 20.8 años ( $D E=2.4$; Mínima $=17$ años, Máxima $=35$ años), de los cuales $133(59 \%)$ eran mujeres. El cuestionario indagó acerca de la religión y la carrera de procedencia de cada estudiante con los resultados descritos en la Tabla 1.
TABLA 1

Distribución muestral por religión y profesión

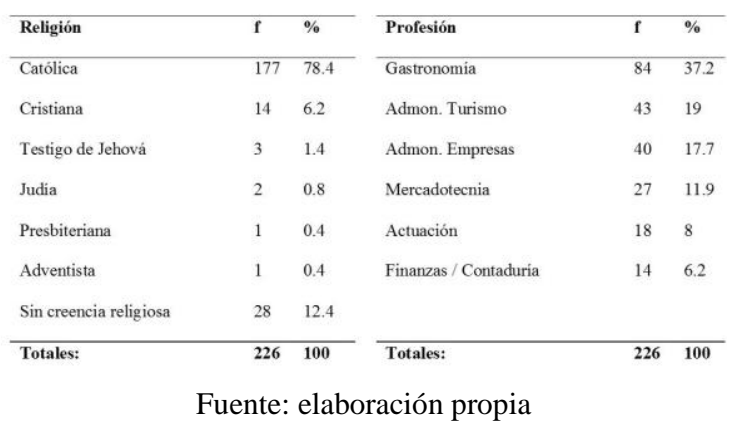

El cuestionario aplicado constó de una primera sección de datos sociodemográficos que incluyó sexo, edad, religión y profesión. Una segunda sección compuesta de cinco preguntas adicionó información asociada a hábitos religiosos; las primeras dos preguntas se enfocaron a hábitos de comunicación con lo divino: "1. ¿Cuántas veces a la semana reza usted?, 2. ¿Cuántas veces a la semana ora usted?". La diferencia entre ambas descansa en los términos rezar y orar; el primero es común y frecuente en algunos contextos (en el catolicismo, por ejemplo), pero inexistente en otros (en religiones como la presbiteriana, cristiana y otras). Estas preguntas se introdujeron como una medida de la importancia que la comunicación con lo divino tiene para el sujeto. En otro sentido, apuntaron la pregunta "3. ¿Cuántas veces al mes (periodo de 4 semanas) va a su iglesia?" que pretendió dar cuenta de la importancia del congregarse con miembros de su iglesia (agrupación formal). La pregunta “4. ¿Cuántas veces a la semana dedica tiempo para el estudio de su religión (leer la Biblia $\mathrm{u}$ otros libros) se centra en la importancia del adoctrinamiento", y la pregunta "5? De los amigos que suele frecuentar durante la semana, ¿qué porcentaje de ellos son importantes porque comparten la misma religión o fe con usted?" (agrupación informal religiosa).

Finalmente, se incluyeron los 12 reactivos de la escala I-E adaptada por Simkin y Etchezahar (2013) en sujetos argentinos. Los reactivos de esta versión fueron evaluados en relación con su correspondencia semántica con respecto al español mexicano; no se identificaron inconsistencias interpretativas que 
reclamasen una adaptación mayor. Como opción de respuesta se empleó una escala Likert con 5 categorías que incluyó en el nivel menor: totalmente en desacuerdo y en el nivel mayor: totalmente de acuerdo, con puntuaciones del 1 al 5 que siguieron ese ordenamiento.

Los cuestionarios fueron aplicados directamente en los salones de clase. A todos los sujetos se les informó acerca del objetivo de la investigación y del carácter anónimo de su participación, destacando que el contestar el cuestionario tenía un carácter voluntario. Todos los sujetos participantes firmaron su consentimiento informado, aplicándose 300 cuestionarios previa exhortación a los alumnos a contestarlos de manera completa; el aplicador revisó cada instrumento resuelto a fin de constatar esta condición. Los cuestionarios fueron analizados con respecto al patrón de respuestas evidenciados en ellos. Conforme a la experiencia obtenida en otros levantamientos de campo, aquellos instrumentos que mostraron un patrón homogéneo de respuesta, es decir, un patrón fijo y repetitivo con respuestas en una sola opción de manera consistente y sostenida en todos los ítems, parecen evidenciar respuestas rápidas e irreflexivas. El eliminar estos cuestionarios suele impactar positivamente la calidad de los datos, lo cual parece evidenciarse en el análisis factorial con estructuras más coherentes. Se descartaron 74 instrumentos homogéneos, seleccionándose 226 cuestionarios heterogéneos útiles (cuestionarios cuyas respuestas no parecen obedecer a un patrón fijo y repetitivo entre las categorías de la escala Likert).

Como era de esperar, la muestra incluyó sujetos religiosos y no religiosos, y para la asignación de los sujetos a tales categorías se decidió aplicar un criterio operacional que en principio exigiría que un sujeto fuese considerado religioso cuando al menos rezara $u$ orara o realizara ambas actividades, al menos 4 veces por semana; que asistiese al menos 4 veces al mes a la iglesia; que al menos $1 \mathrm{vez}$ a la semana estudiase la doctrina de su religión y que al menos el $20 \%$ de sus amigos le resultaran importantes por compartir con él la misma fe.
Sin embargo, de los 226 sujetos solo 7 sujetos cumplieron con este criterio; el resto realizaba las actividades religiosas citadas de forma muy variable, como puede apreciarse en la Tabla 2. Las actividades que parecían ser constantes en un grupo importante de sujetos de toda la muestra eran el rezo y la oración, que al final se eligieron como criterio de clasificación para escoger un total de 78 sujetos "religiosos".

Estos dos comportamientos religiosos parecen constituir los medios usualmente promovidos como ideales para establecer o buscar, generalmente en momentos de solitud, una comunicación con lo divino, lo cual supone la adopción de creencias y apegos religiosos.

\section{TABLA 2}

Distribución de actividades en sujetos religiosos

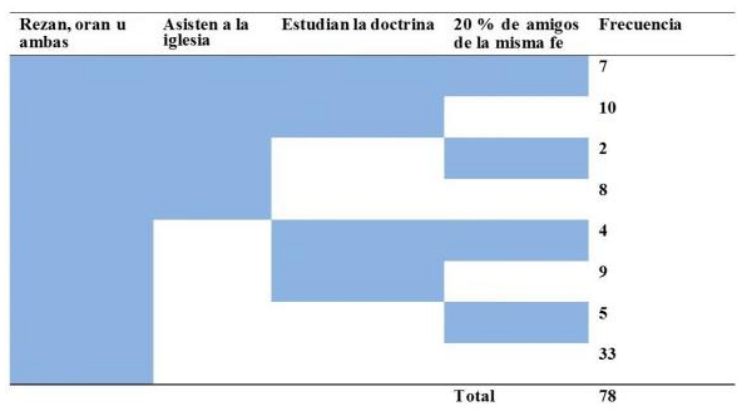

Fuente: elaboración propia

La oración, en sus diferentes tipos, parece estar asociada a la religiosidad intrínseca y contribuir a una mejor calidad de vida (Paloma \& Gallup, 1991 y Paloma \& Pendleton, 1991 citados por Maltby et al., 2010). Maltby et al. (2010) han hallado, al explorar una muestra de sujetos encuestados en iglesias anglicanas, importantes asociaciones directas entre la oración y la religiosidad intrínseca: la oración coloquial, es decir, el platicar con lo divino empleando las propias palabras, la búsqueda de guía, bendiciones, perdón, etc., la oración meditativa, el sentir lo divino, pensar quietamente acerca de lo divino, pasar tiempo adorando a lo divino, etc., la frecuencia de oración, la experiencia de oración, 
entendida como el ser inspirado por lo divino; recibir un insight profundo mediante una verdad espiritual o bíblica, etc., y la oración ritual, es decir, leer un libro de oraciones o recitar oraciones memorizadas, o sea, rezar, $r$. Así, con base en la importancia de la oración y el rezo, fue como se consideró a un sujeto como religioso cuando al menos oraba o rezaba 4 veces por semana.

Los datos fueron capturados y analizados en el sistema SPSS v. 18.0. Se obtuvo un análisis descriptivo de las variables sociodemográficas y de hábitos religiosos. Cada muestra fue evaluada con la medida de adecuación muestral Kaiser-Meyer-Olkin, KMO y la prueba de esfericidad de Bartlett con resultados que favorecieron el análisis factorial exploratorio con rotación VARIMAX y eigenvalues mayores a 1, practicado a cada una de las tres muestras. Se estimó en cada grupo la consistencia interna mediante el alfa de Cronbach. Para las correlaciones se empleó la $r$ de Pearson y para el análisis de diferencias entre medias la prueba $t$ como nivel de significancia se eligió 0.05 o menos y se estimaron intervalos de confianza al $95 \%$.

\section{Resultados}

\section{Validez de constructo}

A fin de evaluar la estructura factorial de la escala en sujetos mexicanos, se decidió explorar tres posibles muestras: una muestra de sujetos religiosos $(N=78)$, otra de sujetos no religiosos $(N=148)$ y una compuesta por todos los sujetos evaluados $(N=226)$, con los resultados que pueden apreciarse en la Tabla 3. Al analizar las tres estructuras resultantes, fue evidente que el mejor resultado se obtuvo en la muestra de sujetos religiosos; aquí fue posible conservar la estructura original del instrumento con excepción del ítem 8, que cargó en dos factores opuestos (intrínseco y extrínseco personal) y del ítem 11 que aportó una carga menor a 0.4 en el factor intrínseco para cargar en el extrínseco personal. Adicionalmente, la estructura de grupos religiosos fue la que explicó el mayor porcentaje de varianza de las tres.

\section{TABLA 3}

Estructura factorial y consistencia interna alfa de Cronbach de la Age Universal I-E Scale en sujetos mexicanos religiosos, no religiosos y mixta

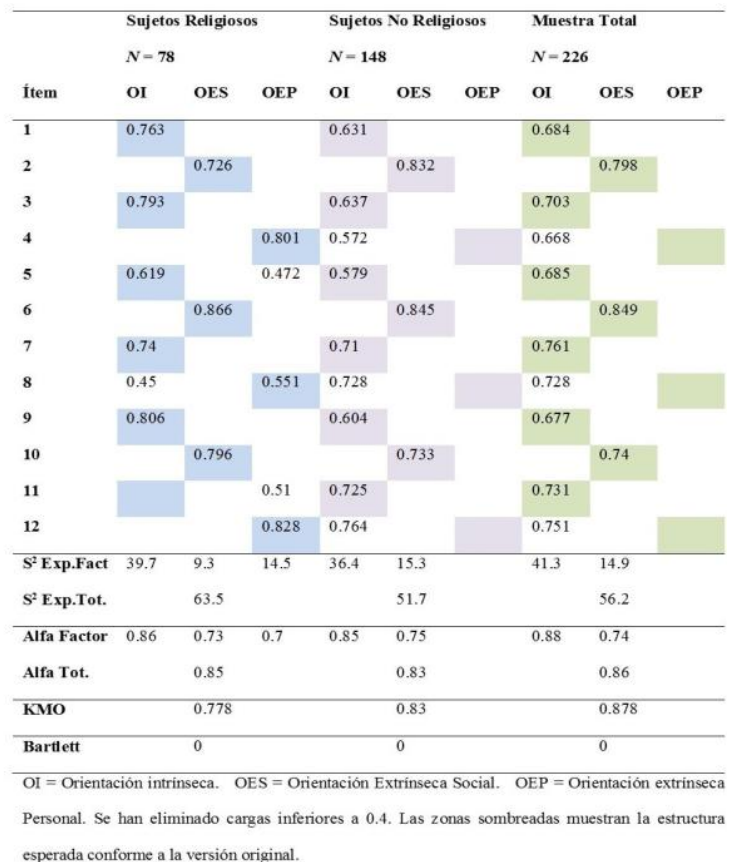

Nota: se han eliminado cargas inferiores a 0.4. Las zonas sombreadas muestran la estructura esperada conforme a la versión original.

Fuente: elaboración propia

\section{Correlación entre subescalas}

Las correlaciones existentes entre la escala total y sus factores muestran altas asociaciones positivas y significativas entre la orientación intrínseca y la extrínseca personal y la escala total; la extrínseca social sin embargo parece correlacionar directa significativa y en una muy menor medida con todas excepto con la escala total, con quien se asocia de manera moderada (Tabla 4). 
Armando Rivera-Ledesma, Sandra Zavala-Jiménez, María Montero-López Lena, Rosa María García-Mendez.

\section{TABLA 4}

Correlación entre las subescalas y la escala total. Estructura de sujetos religiosos

\begin{tabular}{llll}
\hline & O.I. & O.E.S. & O.E.P. \\
\hline O.E.S. & $0.32^{* * * *}$ & \\
O.E.P. & $0.7^{* * *}$ & $0.2^{* * *}$ & \\
Total & $0.92^{* * *}$ & $0.5^{* * *}$ & $0.87^{* * *}$ \\
\hline & O.I. $=$ Orientación intrinseca. & O.E.S. $=$ Orientación extrinseca Social. \\
& O.E.P. $=$ Orientación Extrínseca Personal. * $\leq 0.05, * * \leq 0.01, * * * \leq 0$
\end{tabular}

Fuente: elaboración propia

\section{Consistencia interna}

La consistencia interna del instrumento, evaluada mediante el alfa de Cronbach, fue adecuada en los tres ensayos comparados, ubicándose entre 0.7 y 0.88 para la escala total y sus diferentes factores (Tabla 3 ).

\section{Diferencias entre grupos}

A fin de saber si la escala era apta para discriminar entre sujetos religiosos $\mathrm{y}$ no religiosos, se compararon ambos grupos, considerando la estructura factorial aportada por la muestra de sujetos religiosos (intrínseca, extrínseca social y extrínseca personal). A pesar de que la escala total, el factor de orientación intrínseca y de orientación extrínseca personal discriminaron adecuadamente entre los grupos, aportando diferencias significativas de una magnitud importante, la orientación extrínseca social no fue capaz de ello (Tabla 5).

\section{Discusión}

Los resultados coinciden en buena medida con lo reportado por Simkin y Etchezahar (2013) para la escala I-E-12 explorada en sujetos argentinos. En principio, la escala es semánticamente adecuada para su aplicación en sujetos mexicanos. Del análisis efectuado fue posible descartar inconsistencias en la interpretación de los ítems. Sin embargo, el empleo del término rezar, importante, por ejemplo, en la religión católica, no es común en religiones como la metodista, la presbiteriana o la cristiana, que emplean en su lugar el término oración .

TABLA 5

Análisis de diferencias entre grupos de sujetos. Escala derivada de sujetos religiosos

\begin{tabular}{|c|c|c|c|c|c|c|c|c|c|}
\hline \multirow[b]{2}{*}{ Factor } & \multicolumn{3}{|c|}{ Religiosos } & \multicolumn{3}{|c|}{ No Religiosos } & \multicolumn{3}{|c|}{$\begin{array}{l}\text { Significatividad y tamaño del } \\
\text { efecto }\end{array}$} \\
\hline & $M$ & $D E$ & L.C. $95 \%$ & $M$ & $D E$ & LC. $95 \%$ & $t$ & $d$ & I.C. $95 \%$ \\
\hline o.1. & 15.6 & 5 & $14.5-16.7$ & 11.4 & 4.1 & $10.7-12$ & $6.3^{* * *}$ & 0.91 & $0.62-1.18$ \\
\hline o.E.S. & 4.5 & 2.1 & $4-5$ & 4.3 & 2.2 & $3.9-4.6$ & $0.7 \mathrm{ns.}$ & 0.09 & $0.18-0.36$ \\
\hline O.E.P. & 13.8 & 3.9 & $12.9-14.7$ & 10.1 & 3.7 & $9.5-10.7$ & $6.9^{* * * *}$ & 0.97 & $0.68-1.26$ \\
\hline Total & 34 & 9 & 31.9-36 & 25.9 & 8 & 24.6-27.2 & $6.88^{* * *}$ & 0.96 & $0.92-0.99$ \\
\hline
\end{tabular}

Fuente: elaboración propia

Esta diferencia va más allá de las palabras para significar, en el primer caso, la repetición de un contenido generalmente memorizado y, en el segundo, una activa conversación o adoración enfocada a lo divino (Paloma \& Gallup, 1991 y Paloma \& Pendleton, 1991 citados por Maltby et al., 2010).

Otro término que ha tendido a una modificación persistente en su importancia y significado dentro del contexto de la búsqueda de lo divino en México, es el concepto de religión. La religión se ha ido identificando más con una visión institucional y formal. En su lugar se habla del establecimiento de una relación personal con Dios; del desarrollo de lo espiritual, como resultado de un cambio real en la vida personal hacia los valores demarcados por la religiosidad. Se habla de $f e$, más que de religión. Aun cuando la escala I-E-12 ha continuado siendo investigada y adaptada a distintos contextos culturales (Simkin \& Etchezahar, 2013), parece ser que en la actualidad los constructos que atañen al fenómeno religioso en el campo de las ciencias sociales han sido objeto de una amplia controversia y desarrollo (Rivera-Ledesma, Montero-López, \& Zavala, 2014). La espiritualidad y la religiosidad parecen haber sido asumidas como objetos esenciales de estudio en el campo de la psicología de lo religioso, pero no de manera exclusiva; la espiritualidad existencial como un 
atributo humano no religioso implicado en la vida humana ha sido ampliamente destacada (Paloutzian \& Ellison, 1982).

En el seno de la psicología, más allá de las instituciones y las jerarquías religiosas, son los constructos de religiosidad y espiritualidad los que toman especial interés y relevancia. La religiosidad puede ser comprendida (RiveraLedesma et al., 2014) como un marco conceptual a partir del cual el sujeto puede interpretar lo que ocurre en su relación con lo divino; es también el cuerpo de conocimiento que le permite saber cómo lograr esa relación espiritual, según sea entendida dentro de su contexto social. La religiosidad es un conjunto de cogniciones y conductas definidas por el adoctrinamiento religioso. La espiritualidad es un constructo complejo que, por su parte, siguiendo a Cook (2004 citado por RiveraLedesma et al., 2014), posee tres orientaciones básicas: es intra, inter y trans personal. Intrapersonal, porque favorece la puesta en contacto consigo mismo; interpersonal, porque se alimenta de la identificación que emerge del espejo de la otredad, en el reconocimiento de aquellos a los que se ama y que impregnan la propia visión de la vida en el pasado y el futuro de la existencia y transpersonal, cuando la confrontación se da con lo divino. Es el estar de cara a lo divino... La espiritualidad es un resultado, un efecto relacional, personal, íntimo, y se encuentra lleno de sentido; supone el establecimiento de una relación con lo divino o con el otro; una relación plena de sentido .

La estructura factorial obtenida coincidió en buena medida con la de (Simkin \& Etchezahar, 2013); el $75 \%$ de los reactivos cargaron de manera importante en los factores esperados; dos cargaron en dos factores, uno de esos factores conforme a lo esperado y uno más en un factor distinto. Esto pudo deberse a la muestra de sujetos clasificados como "religiosos"; es probable que un criterio más estricto en el criterio de inclusión empleado para esta categoría (sujetos que recen $\mathrm{u}$ oren al menos 4 veces por semana), redundaría en la obtención de una estructura mejor definida.
Las correlaciones obtenidas entre los tres factores fueron muy similares a los obtenidos por (Simkin \& Etchezahar, 2013), destacando una fuerte asociación positiva y significativa ( $r=$ $0.7, p<0$ ) entre la Intrínseca y la Extrínseca personal, que contrasta con la correlación entre la Intrínseca y extrínseca social $(r=0.32, p<0)$ y la existente entre los dos factores extrínsecos ( $r=0.2, p<0$ ). De acuerdo con estos resultados podría concluirse que no parece encontrarse evidencia que permita afirmar la existencia de un continuum de opuestos entre las orientaciones intrínseca y extrínseca, como Allport y Ross (1967) afirmaron inicialmente; al parecer, en muestras compuestas por sujetos religiosos los factores tenderían a ser independientes en sujetos latinos.

La consistencia interna fue muy similar a la obtenida en sujetos argentinos para las tres subescalas, identificando diferencias en la varianza explicada en sujetos mexicanos vs. sujetos argentinos (respectivamente: Total: 63.5 \% vs. $70.3 \%$; Intrínseca: $39.7 \%$ vs. $28.8 \%$; Extrínseca social: $9.3 \%$ vs. $22.7 \%$; Extrínseca personal: $14.5 \%$ vs. $18.8 \%$ ). Finalmente, los resultados permiten añadir evidencia a la validez discriminante de la Escala I-E-12 al ser capaz de discriminar claramente entre sujetos religiosos y no religiosos, mediante los factores Intrínseco y Extrínseco personal. Sin embargo, la extrínseca social no fue capaz de discriminar entre sujetos religiosos y no religiosos; este factor es también el más estable entre las tres estructuras factoriales calculadas; mantiene su estructura y cargas muy similares en las tres muestras. Quizá resulte favorecedor explorar la conveniencia de mejorar la idoneidad conceptual de los ítems de este factor.

Una de las contribuciones importantes de la escala propuesta originalmente por Allport y Ross (1967) fue justamente el llamar la atención sobre el verdadero sentido de la vida religiosa; establecer una relación personal con lo divino. Diferenciar entre lo utilitario y la experiencia transformadora de la fe. En la actualidad, hablar de religiosidad intrínseca supone el hablar de vida espiritual, y es posible que la orientación extrínseca sea más inteligible 
si se le conceptualiza como un tipo de estrategia de afrontamiento religioso, es decir, como la traducción que hace una persona de su búsqueda de lo divino en conductas, cogniciones y afectos.

\section{Referencias}

Allport, G. W., \& Ross, J. M. (1967). Personal religious orientation and prejudice. Journal of Personality and Social Psychology , 5 , 432-443. http://dx.doi.org/10.1037/h00212 12

Burris, C. T. (1999). Religious Orientation Scale. En P. Hill \& R. W. Hood (Eds.), Measures of religiosity (pp. 144-154). Birmingham, AL: Religious Education Press.

Gorsuch, R. L., \& Venable, G. D. (1983). Development of an Age Universal I-E Scale. Journal for the Scientific Study of Religion, 22 , 181-187. http://dx.doi.org/10 $.2307 / 1385677$

Kirkpatrick, L. A. (1989). A psychometric analysis of the Allport-Ross and Feagan measures of intrinsic-extrinsic religious orientation. En M. L. Lynn \& D. O. Moberg (Eds.), Research in the social scientific study of religion (Vol.1, pp. 1-30). Greenwich, CT: JAI Press.

Leong, F. T., \& Zachar, P. (1990). An evaluation of Allport's Religious Orientation Scale across one Australian and two United States samples. Educational and Psychological Measurement, 50, 359-368. http://dx.doi.org/10.1177/001316 4490502014

Maltby, J. (1999). The internal structure of a derived, revised, and amended measure of the Religious Orientation Scale: The AgeUniversal I-E Scale-12. Social Behaviour and Personality, 27 , 407-412. http://dx.do i.org/10.2224/sbp.1999.27.4.407

Maltby, J. (2002). The Age Universal IE Scale 12 and orientation toward religion: Confirmatory factor analysis. The Journal of Psychology: Interdisciplinary and Applied, 136 (5), 555-560. http://dx.do i.org/10.1080/00223980209605550
Maltby, J., Lewis, C. A., Freeman, A., Day, L., Cruise, S. M., \& Breslin, M. J. (2010). Religion and health: The application of a cognitive behavioural framework. Mental Health, Religion and Culture, 13 (7-8), 749-759.

Núñez-Alarcón, M., Moreno-Jiménez, M. P., \& Moral-Toranzo, F. (2011). Modelo causal del prejuicio religioso. Anales de Psicología, 27 (3), 852-861.

Paloutzian, R., \& Ellison, C. (1982). Loneliness, spiritual well-being and the quality of life. En L. Peplau \& D. Perlman (Eds.), Loneliness. A sourcebook of current theory, research and therapy (pp. 224-237). New York: John Wiley \& Sons.

Rivera-Ledesma, A., Montero-López Lena, M., \& Zavala, J. S. (2014). Espiritualidad, psicología y salud. Psicología y Salud, 24 (1), 139-152.

Simkin, H., \& Etchezahar, E. (2013). Las orientaciones religiosas extrínseca e intrínseca: validación de la "Age Universal" I-E Scale en el contexto argentino. Psykhe, 22 (1), 97-106.

\section{Notas}

\section{* Artículo de investigación.}

